\title{
Supplemental dietary nitrate for chronic obstructive pulmonary disease: a randomized, double-blind, placebo-controlled, crossover trial
}

\author{
C. P. Kerley ${ }^{1,2}$, K. Cahill ${ }^{1}$, K. Bolger ${ }^{1}$, K. Fennell ${ }^{1}$, A. O’Brien ${ }^{1}$, A. McGowan ${ }^{1}$, C. Burke ${ }^{1}$, \\ J. L. Faul ${ }^{1}$ and L. J. Cormican ${ }^{1}$ \\ ${ }^{1}$ Respiratory \& Sleep Diagnostics Department, Connolly Hospital, Blanchardstown, Dublin 15, Republic of Ireland and \\ ${ }^{2}$ University College Dublin, Belfireld, Dublin 4, Republic of Ireland
}

Nitric oxide (NO) is an important systemic and pulmonary arterial vasodilator. The conversion of nitrite (derived from dietary nitrate) to nitric oxide can occur independent of nitric oxide synthase (Lundberg \& Govoni 2004) in a process that is upregulated in hypoxic conditions (Lundberg et al., 2008). Since patients with chronic obstructive pulmonary disease (COPD) commonly suffer hypoxaemia during exercise, we hypothesized that dietary nitrate supplementation might acutely improve exercise capacity in hypoxic COPD patients through enhanced production of NO.

We compared the acute effect of beetroot juice (containing $14 \mathrm{mmol}$ of nitrate) on exercise capacity and arterial systolic blood pressure in COPD patients compared to a matched placebo drink (containing less than $0.5 \mathrm{mmol}$ of dietary nitrate).

Twelve subjects (6 male) with COPD were recruited. Resting blood pressure was assessed in duplicate (manual sphygmomanometer). Heart rate and arterial oxygen concentration (pulse oximetry) as well as self-reported breathlessness (Borg dyspnea scale) were assessed pre- and post-incremental shuttle walk test. Subjects were then randomized to drink beetroot juice or a matched placebo and the protocol was repeated 3 hours later. The $3 \mathrm{~h}$ rest period was to allow for sufficient recovery from baseline testing and has been found to correspond with peak plasma nitrite concentrations following oral consumption of dietary nitrate (Webb et al., 2008). After a 7-day washout period, the protocol was repeated with the crossover beverage.

There were no significant differences in heart rate, arterial oxygen concentrations, or breathlessness at any time point. However, COPD subjects who took dietary nitrate walked significantly further than when they took placebo $(+23 v s$. -13 metres; $p<0.01)$ and had a reduction in both systolic blood pressure $(-13 v s .0 \mathrm{mmHg} ; p<0.05)$ and diastolic blood pressure $(-3.2 \mathrm{vs} .+7.8 \mathrm{mmHg} ; p<0.05)$.

Acute consumption of dietary nitrate can improve exercise tolerance and lower blood pressure in COPD patients.

1. Lundberg JO, Govoni M (2004) Free Radic Biol Med 37, 395-400.

2. Lundberg JO, Weitzberg E, Gladwin MT (2008) Nat Rev Drug Discov 7, 156-67.

3. Webb AJ, Patel N, Loukogeorgakis S, et al. (2008) Hypertension. 51, 784-90. 